Check for updates

Cite this: Chem. Commun., 2020, 56,6420

Received 31st March 2020,

Accepted 30th April 2020

DOI: $10.1039 / \mathrm{dOcc02321c}$

rsc.li/chemcomm

\section{Chemical synthesis of human trefoil factor 1 (TFF1) and its homodimer provides novel insights into their mechanisms of action $\dagger$}

\author{
Nayara Braga Emidio, (D) ${ }^{a}$ Hayeon Baik, ${ }^{b}$ David Lee, ${ }^{a}$ René Stürmer, ${ }^{c}$ Jörn Heuer, ${ }^{c}$ \\ Alysha G. Elliott, (D) a Mark A. T. Blaskovich, (D) ${ }^{a}$ Katharina Haupenthal, ${ }^{c}$ \\ Nicole Tegtmeyer, ${ }^{d}$ Werner Hoffmann, ${ }^{c}$ Christina I. Schroeder (DD ${ }^{\text {ae }}$ and \\ Markus Muttenthaler (D) *ab
}

TFF1 is a key peptide for gastrointestinal protection and repair. Its molecular mechanism of action remains poorly understood with synthetic intractability a recognised bottleneck. Here we describe the synthesis of TFF1 and its homodimer and their interactions with mucins and Helicobacter pylori. Synthetic access to TFF1 is an important milestone for probe and therapeutic development.

TFF1 is a member of the trefoil factor family (TFF) of gastrointestinal peptides, well-known for their role in protecting and repairing the gastrointestinal tract. ${ }^{1-3}$ A substantial body of evidence supports TFF1's role in gastric protection. ${ }^{1-5}$ TFF1 is typically expressed in gastric surface mucous cells ${ }^{3}$ and TFF1 knockout $\left(^{-/}\right)$mice spontaneously develop antropyloric adenoma with about $30 \%$ progressing to carcinomas, establishing TFF1 as a tumour suppressor. ${ }^{6,7}$ By contrast, transgenic mice overexpressing TFF1 display increased resistance to gastrointestinal damage ${ }^{8}$ and TFF1 is ectopically expressed in an in vivo model of acetic acid-induced colitis. ${ }^{9}$ Administration of TFF1 has additional protective and healing effects in animal models of gastrointestinal injury. ${ }^{10-12}$ Consequently, TFF1 has therapeutic potential for gastrointestinal disorders including inflammatory bowel diseases (IBD) and non-steroidal anti-inflammatory drug (NSAID)-induced gastritis. $^{13-16}$

TFF1 is a 60 residue peptide with three disulfide bonds $\left(\mathrm{Cys}^{\mathrm{I}-\mathrm{V}}\right.$, Cys ${ }^{\mathrm{II}-\mathrm{IV}}$, Cys $\left.{ }^{\mathrm{II}-\mathrm{VI}}\right)$ resulting in a highly structured and conserved three-loop containing TFF domain. ${ }^{1,17}$ A free cysteine

\footnotetext{
${ }^{a}$ Institute for Molecular Bioscience, The University of Queensland, St Lucia, Brisbane, Queensland, 4072, Australia

${ }^{b}$ Institute of Biological Chemistry, Faculty of Chemistry, University of Vienna, Währingerstr. 38, Vienna, 1090, Austria.

E-mail:markus.muttenthaler@univie.ac.at

${ }^{c}$ Institute of Molecular Biology and Medicinal Chemistry, Otto-von-GuerickeUniversity Magdeburg, Leipziger Str. 44, 39120 Magdeburg, Germany

${ }^{d}$ Division of Microbiology, Department of Biology, Friedrich-Alexander-University, Erlangen-Nürnberg Staudtstr. 5, 91058 Erlangen, Germany

${ }^{e}$ National Cancer Institute, National Institutes of Health, Frederick, MD, 21702, USA

† Electronic supplementary information (ESI) available. See DOI: 10.1039/ d0cc02321c
}

residue (Cys ${ }^{\mathrm{VII}}$ ) is located outside the TFF domain and enables homo- and heterodimerisation via an intermolecular disulfide bond (e.g. TFF1-gastrokine 2). ${ }^{5,18,19}$ The molecular mechanisms through which TFF1 protects the gastrointestinal mucosa are not fully understood, ${ }^{10,11}$ but could involve enhancement of cell migration due to its motogenic ${ }^{20}$ and anti-apoptotic ${ }^{21}$ effects ('restitution'), ${ }^{22}$ a scavenger function for extracellular reactive oxygen/nitrogen species, ${ }^{5}$ and/or mucin interactions. ${ }^{3,5}$ Lack of synthetic access to TFF1 and associated probes is the main bottleneck hindering progress on identification of its mode of action on a molecular level. Recombinant expression and purification of TFF1 is challenging, ${ }^{18,23}$ and synthesis of TFF1 monomer was attempted in the past, but no correctly folded TFF1 was obtained nor characterised. ${ }^{24}$ Reliable access to TFF1 peptides through chemical synthesis would facilitate large scale production, library design for structure-activity relationship (SAR) studies and site-specific chemical modifications for molecular probes, setting the stage for therapeutic development. We thus set out to develop a synthetic strategy for the efficient production of bioactive TFF1 and its analogues.

Attempts to directly assemble TFF1 via solid phase peptide synthesis (SPPS) were unsuccessful and prompted us to switch to a two-fragment ligation strategy (Fig. 1). TFF1 was split between Gly $^{31}$ and $\mathrm{Cys}^{32}$ which was chosen based on fragment size and favoured kinetics for native chemical ligation (NCL) (ligation proceeds faster at sterically less hindered thioester positions). ${ }^{25,26}$ Traditionally, thioester fragments are synthesised by tert-butyloxycarbonyl (Boc)-SPPS because the thioester is not stable under the basic conditions and nucleophiles required for $\mathrm{N}^{\alpha}$-Fmoc deprotection. ${ }^{27}$ Attempts to synthesise the $\mathrm{N}$-terminal segment containing the thioester by manual Boc-SPPS were however unsuccessful, resulting in poor crude quality and low yield. Thus, a Fmoc-SPPS compatible strategy that uses a C-terminal hydrazide as a thioester surrogate was pursued (Fig. 1). ${ }^{28}$ TFF 1 1-31 $_{1}$ was produced with a C-terminal hydrazide that was converted to a thioester prior to ligation with $\mathrm{TFF}_{32-60}$ to form full-length reduced $\mathrm{TFF} 1_{1-60}$ (Fig. $2 \mathrm{~A}$ and $\mathrm{B}$ ). $\mathrm{TFF}_{1-60}$ was then folded at $\mathrm{pH} 8.5(50 \mu \mathrm{M})$ for 48 hours, forming 

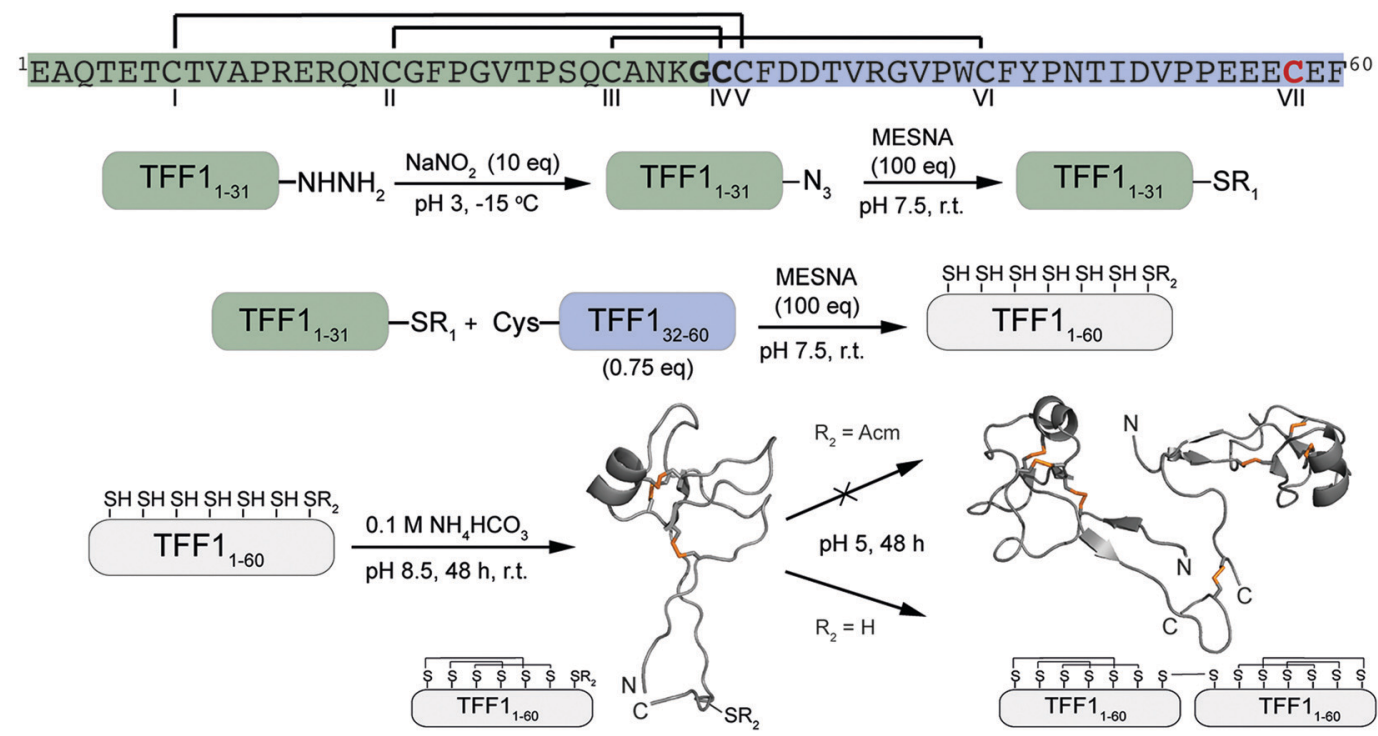

Fig. 1 Synthetic strategy of the assembly of TFF1 monomer and homodimer. TFF1 sequence highlighted in green represents the N-terminal fragment and in blue the C-terminal fragment used for native chemical ligation. Residues in bold highlight the ligation site. $C^{\text {VII }}$ (red) enables dimerisation. Disulfide connectivity is indicated with black lines. NMR structures used for this scheme: TFF1 monomer PDB: 1PS2; TFF1 homodimer PDB: 1HI7. Disulfide bonds are shown in orange.

three disulfide bonds. TFF1 displayed a two-peak analytical HPLC profile with identical mass (Fig. 2C). Both peaks were collected and each re-run, yielding the same two-peak profile, confirming a single molecular entity. Such multi-peak conformational HPLC peak profiles are not uncommon with peptides and proteins $^{29-32}$ and have also been observed with TFF3. ${ }^{33}$

Homodimerisation of TFF1 was achieved through disulfide bond formation in water at high concentration $(1.5 \mathrm{mM}$; $\mathrm{pH} \sim 5,48 \mathrm{~h})$. TFF1 homodimer also displayed a two-peak HPLC profile (Fig. 2D) with identical mass. Since TFF1 dimerises slowly in water, we also synthesised TFF1 with Cys ${ }^{\mathrm{VII}}$ protected (TFF1 $\left.\left(\mathrm{C}^{58} \mathrm{Acm}\right)\right)$ to ensure proper differentiation between TFF1
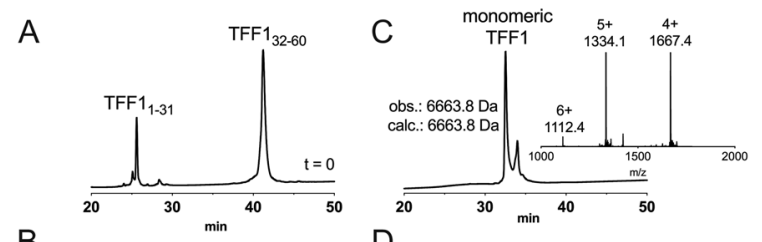

B
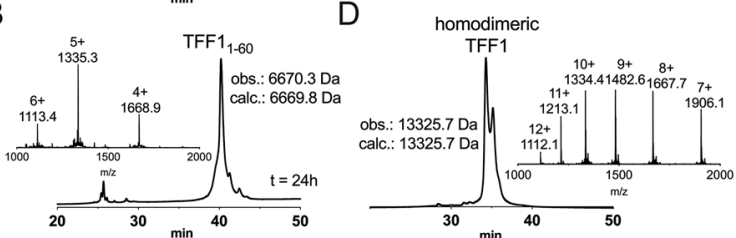

Fig. 2 Chemical synthesis of TFF1 and TFF1 homodimer. (A) Analytical $\mathrm{C}_{18}$-RP-HPLC trace of $\mathrm{NCL}$ at $\mathrm{O}$ h. NCL occurred after the hydrazide fragment was converted into a thioester peptide through in situ activation and thiolysis. (B) Analytical $\mathrm{C}_{18}-\mathrm{RP}-\mathrm{HPLC}$ and MS traces of NCL at $24 \mathrm{~h}$. (C) Analytical $\mathrm{C}_{3}$-RP-HPLC and MS traces of folded TFF1. Reduced fulllength TFF1 was oxidised in $0.1 \mathrm{M} \mathrm{NH}_{4} \mathrm{HCO}_{3}, \mathrm{pH} 8.5$ for $48 \mathrm{~h}$. (D) Analytical $\mathrm{C}_{3}$-RP-HPLC and MS traces of folded TFF1 homodimer. TFF1 was dimerised over $48 \mathrm{~h}$ in water. monomer and homodimer for our functional studies (Fig. S1, ESI $\dagger$ ) and to have the option to unprotect $\mathrm{Cys}^{\mathrm{VII}}$ when needed for dimer formation or conjugation to reporter tags. In previous studies with recombinant TFF1, undesired homodimerisation was achieved through a Cys ${ }^{\mathrm{VII}}$ to Ser replacement. ${ }^{20}$

NMR spectroscopy confirmed the correct fold of both TFF1 and TFF1 homodimer. $\mathrm{H} \alpha$ chemical shifts were assigned using total correlated spectroscopy (TOCSY) and nuclear Overhauser effect spectroscopy (NOESY) and compared to the chemical shifts of recombinant TFF1 (BMRB: 4933$)^{34}$ and TFF1 homodimer (BMRB: 4930). ${ }^{23}$ Synthetic and recombinant TFF1 analogues displayed good overlap of the secondary $\mathrm{H} \alpha$ chemical shifts (deviation of the $\mathrm{H} \alpha$ chemical shifts from random coil values $^{35}$ ) confirming the same overall fold and $3 \mathrm{D}$ structure (Fig. 3). Circular dichroism (CD) experiments confirmed the presence of an $\alpha$-helix characterised by negative bands at $208 \mathrm{~nm}$ and $222 \mathrm{~nm}$ and a positive band at $193 \mathrm{~nm}$ (Fig. S2, $\mathrm{ESI}+$ ), corresponding well with the presence of negative secondary $\mathrm{H} \alpha$ chemical shifts indicative of an $\alpha$-helix between position Pro $^{24}-$ Lys $^{30}$ in loop 2 (Fig. 3)..$^{23,34}$

Interaction of TFF1 $\left(\mathrm{C}^{58} \mathrm{Acm}\right)$ and TFF1 homodimer with carbohydrates was tested in different mucin binding assays since TFF1 has been associated with lectin activities. ${ }^{36-39}$ Both peptides were labelled with ${ }^{125} \mathrm{I}^{40}$ and then used in binding studies against purified mucin fractions from the stomach of human, ${ }^{41}$ pig $^{40}$ and frog $^{42}$ (Xenopus laevis) (Fig. 4).

In these overlay assays, only TFF1 homodimer bound to these mucins. Attempts to bind TFF $1\left(\mathrm{C}^{58} \mathrm{Acm}\right)$ to mucin preparations failed. This indicates that dimerisation of TFF1 is essential for mucin interaction. This is in agreement with other observations that have also highlighted dimerisation as a critical factor for the protective function of TFF1. ${ }^{43,44}{ }^{125}$ I-TFF1 homodimer also bound to a reduced porcine mucin preparation 


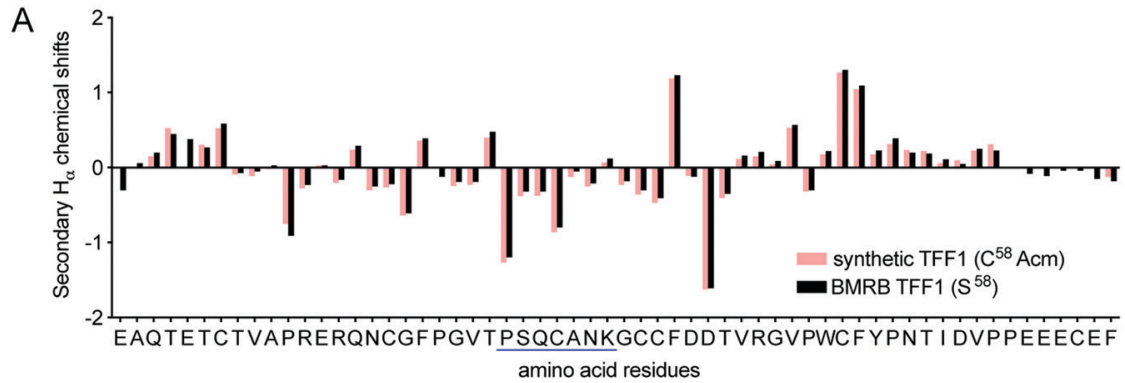

B

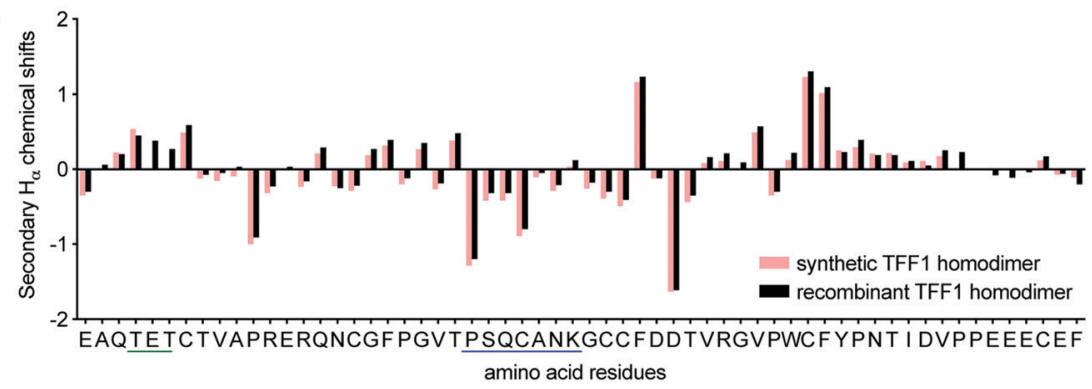

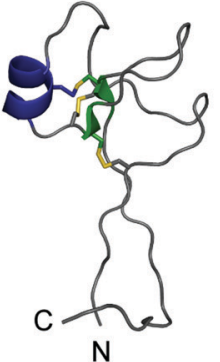

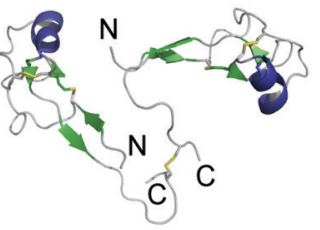

Fig. 3 Structural secondary $\mathrm{H} \alpha$ chemical shift comparison of synthetic and recombinant TFF1 by NMR. (A) Comparison of synthetic TFF1(C $\left.{ }^{58} \mathrm{Acm}\right)$ and recombinant TFF1 $\left(S^{58}\right)$ (BMRB: 4933). ${ }^{34}$ (B) Comparison of synthetic TFF1 and recombinant TFF1 homodimer (BMRB: 4930). ${ }^{23}$ A putative $\alpha$-helix is highlighted in blue in the sequence and NMR structure (TFF1 monomer PDB: 1PS2; TFF1 homodimer PDB: 1HI7).

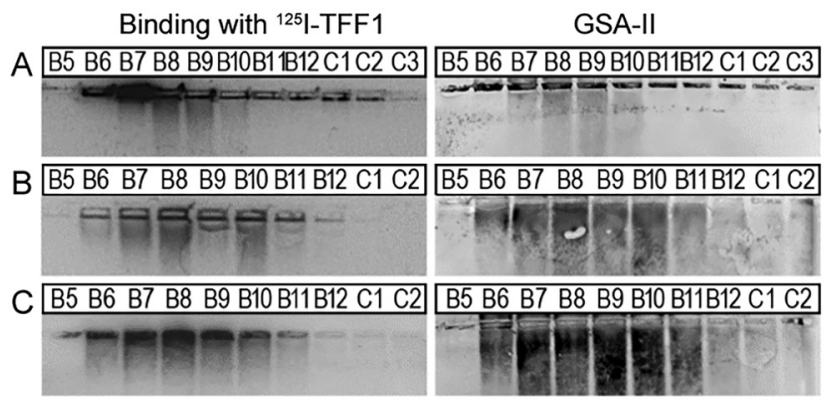

Fig. 4 Binding studies of ${ }^{125}$ I-TFF1 homodimer with gastric mucins Autoradiography was obtained after incubating ${ }^{125}$ I-TFF1 homodimer with different fractions purified (B5-C2/C3) by size exclusion chromatography from (A) human gastric mucins, ${ }^{41}$ (B) reduced porcine gastric mucins, ${ }^{40}$ and $(C)$ gastric mucins from $X$. laevis. ${ }^{42}$ These fractions were separated by non-denaturing agarose gel electrophoresis which was directly blotted on a nitrocellulose membrane. Reactivity with GSA-II, a lectin from Griffonia simplicifolia, confirms the presence of mucins since it recognises $\alpha \mathrm{GlcNAc}$ ( $\alpha 1,4$-linked $N$-acetylglucosamine), a conserved carbohydrate motif in gastric mucins.

(boiled with $1 \% \beta$-mercaptoethanol) (Fig. 4B), suggesting lectinlike binding to the carbohydrate moieties and not to its protein structure. ${ }^{36-38}$ Binding to frog mucins demonstrated that the gastric mucin sugar epitope recognised by TFF1 is evolutionary conserved (Fig. 4C). GSA-II, a lectin from Griffonia simplicifolia that recognises $\alpha$ GlcNAc $(\alpha 1,4$-linked $N$-acetylglucosamine; a conserved carbohydrate motif in gastric mucins), confirmed the presence of mucins. As a positive control and proof of synthetic TFF1 homodimer lectin activity, binding with cell lysates of $H$. pylori was tested (Fig. 5). A clear positive signal with the lipopolysaccharide was obtained with the $H$. pylori wild type in agreement with previous reports. ${ }^{36-39}$ In contrast, no binding was observed with a $H$. pylori mutant lacking seduheptulose 7-phosphate isomerase (P12 $\triangle H P 0857)$ which results in a truncated lipopolysaccharide core oligosaccharide. ${ }^{45}$

TFF1 has also been implicated in cell migration and proliferation. ${ }^{3,20,21}$ However, independent confirmation, target identification and in-depth pharmacology remain sparse for these studies. ${ }^{1}$ With multi-milligrams of TFF1 at hand, our goal was to validate some of these studies in well-controlled experiments. We carried out cell migration and proliferation assays with HT-29 (human colorectal adenocarcinoma) cells using a state-of-the-art IncuCyte ${ }^{\mathbb{R}}$ system. In these studies, neither TFF1 nor TFF1 homodimer had any effect on cell migration or proliferation at concentrations of $0.1-10 \mu \mathrm{M}$ (Fig. S3, ESI $\dagger$ ). We also performed toxicity assays with TFF1 and its homodimer. Neither peptide

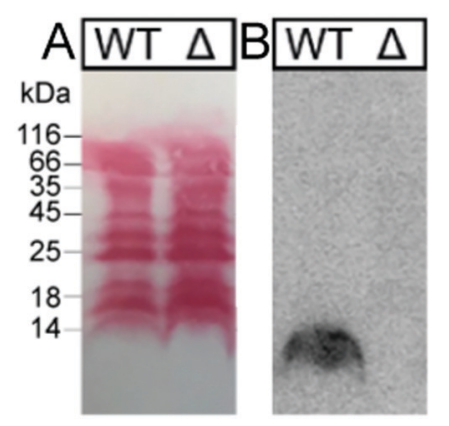

Fig. 5 Binding studies of $H$. pylori extracts with ${ }^{125}$ I-TFF1 homodimer. $H$. pylori cell lysates (WT: wild type P12; $\Delta$ : mutant P12 $\Delta H P 0857$ ) were run on a $15 \%$ SDS-PAGE and blotted on a nitrocellulose membrane. (A) Protein staining with Ponceau $S$ showed that the mutation did not alter total protein expression. (B) Incubation of ${ }^{125}$ I-TFF1 with $H$. pylori cell lysates and autoradiography showed binding to WT but no binding to $H$. pylori mutant with a truncated lipopolysaccharide core oligosaccharide. 
displayed any cytotoxic or haemolytic effects at concentrations up to $25 \mu \mathrm{M}$ (Fig. S4, ESI $\dagger$ ).

In conclusion, we developed a synthetic strategy for TFF1, its homodimer and analogues. TFF1 homodimer bound to gastric mucins supporting the consensus that TFF1 elicits its protective action in the gut via lectin-like mucin cross-linking. We could not reproduce TFF1 activity in well-controlled HT-29 cell migration or proliferation assays (see ESI $\dagger$ for further discussion). Chemical access to TFF1 and its analogues represents an important milestone for the TFF field since it enables the production of homogenous material in addition to regioselective incorporation of chemical modifications that will facilitate molecular probe development for more in-depth mechanistic and target validation studies as well as therapeutic development. This in turn will advance our understanding of TFF1's physiology and therapeutic potential.

M. M. was supported by the European Research Council under the European Union's Horizon 2020 Research and Innovation Program (714366) and by the Australian Research Council (DE150100784, DP190101667). C. I. S. was an ARC Future Fellow (FT160100055). N. B. E. was supported by the University of Queensland International Postgraduate Scholarship. We thank Janet Reid and CO-ADD (the Community for Open Antimicrobial Drug Discovery), funded by the Wellcome Trust (Strategic Award $104797 / Z / 14 / Z$ ) and the University of Queensland, for the cytotoxicity and haemolysis assays. We thank Prof. S. Backert (FAU Erlangen-Nürnberg) and Prof. Paul Alewood (The University of Queensland) for their support of this work.

\section{Conflicts of interest}

There are no conflicts to declare.

\section{Notes and references}

1 N. Braga Emidio, W. Hoffmann, S. M. Brierley and M. Muttenthaler, Trends Biochem. Sci., 2019, 44, 387-390.

2 D. Taupin and D. K. Podolsky, Nat. Rev. Mol. Cell Biol., 2003, 4, 721-732.

3 S. Ribieras, C. Tomasetto and M. C. Rio, Biochim. Biophys. Acta, Rev. Cancer, 1998, 1378, F61-F77.

4 E. B. Znalesniak, F. Salm and W. Hoffmann, Int. J. Mol. Sci., 2020, 21, 644-659.

5 J. Heuer, F. Heuer, R. Stürmer, S. Harder, H. Schlüter, N. Braga Emidio, M. Muttenthaler, D. Jechorek, F. Meyer and W. Hoffmann, Int. J. Mol. Sci., 2020, 21, 2508-2526.

6 O. Lefebvre, M. P. Chenard, R. Masson, J. Linares, A. Dierich, M. LeMeur, C. Wendling, C. Tomasetto, P. Chambon and M. C. Rio, Science, 1996, 274, 259-262.

7 C. Tomasetto and M. C. Rio, Cell. Mol. Life Sci., 2005, 62, 2916-2920.

8 R. J. Playford, T. Marchbank, R. A. Goodlad, R. A. Chinery, R. Poulsom, A. M. Hanby and N. A. Wright, Proc. Natl. Acad. Sci. U. S. A., 1996, 93, 2137-2142.

9 H. Itoh, M. Tomita, H. Uchino, T. Kobayashi, H. Kataoka, R. Sekiya and Y. Nawa, Biochem. J., 1996, 318, 939-944.

10 A. J. FitzGerald, M. Pu, T. Marchbank, B. R. Westley, F. E. B. May, J. Boyle, M. Yadollahi-Farsani, S. Ghosh and R. J. Playford, Peptides, 2004, 25, 793-801.

11 K. Vandenbroucke, W. Hans, J. Van Huysse, S. Neirynck, P. Demetter, E. Remaut, P. Rottiers and L. Steidler, Gastroenterology, 2004, 127, 502-513.
12 S. Caluwaerts, K. Vandenbroucke, L. Steidler, S. Neirynck, P. Vanhoenacker, S. Corveleyn, B. Watkins, S. Sonis, B. Coulie and P. Rottiers, Oral Oncol., 2010, 46, 564-570.

13 N. A. Wright, Gut, 2001, 48, 293-294.

14 C. Tomasetto, R. Masson, J. L. Linares, C. Wendling, O. Lefebvre, M. P. Chenard and M. C. Rio, Gastroenterology, 2000, 118, 70-80.

15 J. L. Newton, A. Allen, B. R. Westley and F. E. B. May, Gut, 2000, 46, 312-320.

16 C. Dunne, J. Naughton, G. Duggan, C. Loughrey, M. Kilcoyne, L. Joshi, S. Carrington, H. Earley, S. Backert, C. R. Masselot, F. E. B. May and M. Clyne, Microorganisms, 2018, 6, 44-58.

17 L. Thim and F. E. B. May, Cell. Mol. Life Sci., 2005, 62, 2956-2973.

18 M. P. Chadwick, B. R. Westley and F. E. B. May, Biochem. J., 1997, 327, 117-123.

19 B. R. Westley, S. M. Griffin and F. E. B. May, Biochemistry, 2005, 44, 7967-7975.

20 T. Marchbank, B. R. Westley, F. E. B. May, D. P. Calnan and R. J. Playford, J. Pathol., 1998, 185, 153-158.

21 C. Bossenmeyer-Pourie, R. Kannan, S. Ribieras, C. Wendling, I. Stoll, L. Thim, C. Tomasetto and M. C. Rio, J. Cell Biol., 2002, 157, 761-770.

22 W. Hoffmann, Cell. Mol. Life Sci., 2005, 62, 2932-2938.

23 M. A. Williams, B. R. Westley, F. E. B. May and J. Feeney, FEBS Lett., 2001, 493, 70-74.

24 P. D. Rye, J. Keyte, M. J. Sutcliffe, K. Bailey and R. A. Walker, Protein Pept. Lett., 1994, 1, 54-59.

25 E. C. B. Johnson and S. B. H. Kent, J. Am. Chem. Soc., 2006, 128, 6640-6646.

26 T. M. Hackeng, J. H. Griffin and P. E. Dawson, Proc. Natl. Acad. Sci. U. S. A., 1999, 96, 10068-10073.

27 M. Muttenthaler, F. Albericio and P. E. Dawson, Nat. Protoc., 2015, 10, 1067-1083.

28 J.-S. Zheng, S. Tang, Y.-K. Qi, Z.-P. Wang and L. Liu, Nat. Protoc., 2013, 8, 2483-2495.

29 H. B. Luo, M. Y. Cao, K. Newell, C. Afdahl, J. Wang, W. K. Wang and Y. L. Li, J. Chromatogr. A, 2015, 1424, 92-101.

30 F. C. Cardoso, Z. Dekan, J. J. Smith, J. R. Deuis, I. Vetter, V. Herzig, P. F. Alewood, G. F. King and R. J. Lewis, Br. J. Pharmacol., 2017, 174, 2528-2544.

31 J. S. Wingerd, C. A. Mozar, C. A. Ussing, S. S. Murali, Y. K. Chin, B. Cristofori-Armstrong, T. Durek, J. Gilchrist, C. W. Vaughan, F. Bosmans, D. J. Adams, R. J. Lewis, P. F. Alewood, M. Mobli, M. J. Christie and L. D. Rash, Sci. Rep., 2017, 7, 974-988.

32 H. Takahashi, J. I. Kim, H. J. Min, K. Sato, K. J. Swartz and I. Shimada, J. Mol. Biol., 2000, 297, 771-780.

33 L. Thim, H. F. Woldike, P. F. Nielsen, M. Christensen, K. Lynchdevaney and D. K. Podolsky, Biochemistry, 1995, 34, 4757-4764.

34 M. D. Carr, Biochemistry, 1992, 31, 1998-2004.

35 D. S. Wishart, C. G. Bigam, A. Holm, R. S. Hodges and B. D. Sykes, J. Biomol. NMR, 1995, 5, 67-81.

36 M. Clyne, P. Dillon, S. Daly, R. O'Kennedy, F. E. May, B. R. Westley and B. Drumm, Proc. Natl. Acad. Sci. U. S. A., 2004, 101, 7409-7414.

37 E. P. Reeves, T. Ali, P. Leonard, S. Hearty, R. O'Kennedy, F. E. May, B. R. Westley, C. Josenhans, M. Rust, S. Suerbaum, A. Smith, B. Drumm and M. Clyne, Gastroenterology, 2008, 135, 2043-2054.

38 B. Dolan, J. Naughton, N. Tegtmeyer, F. E. B. May and M. Clyne, PLoS One, 2012, 7, e47300.

39 M. Clyne and F. E. B. May, Int. J. Mol. Sci., 2019, 20, 4400-4413.

40 R. Stürmer, S. Harder, H. Schlüter and W. Hoffmann, ChemBioChem, 2018, 19, 2598-2608.

41 F. Heuer, R. Stürmer, J. Heuer, T. Kalinski, A. Lemke, F. Meyer and W. Hoffmann, Int. J. Mol. Sci., 2019, 20, 871-5883.

42 R. Stürmer, J. Reising and W. Hoffmann, Int. J. Mol. Sci., 2019, 20, 6052-6060.

43 F. G. Hanisch, H. Ragge, T. Kalinski, F. Meyer, H. Kalbacher and W. Hoffmann, Glycobiology, 2013, 23, 2-11.

44 L. Thim, F. Madsen and S. S. Poulsen, Eur. J. Clin. Invest., 2002, 32, 519-527.

45 C. K. Yu, C. J. Wang, Y. Chew, P. C. Wang, H. S. Yin and M. C. Kao, Biochem. Biophys. Res. Commun., 2016, 477, 794-800. 